\title{
Performance of Immunoassay Kits for Site Characterization and Remediation
}

\author{
Larry C. Waters, Andi Palausky, Richard W. Counts, and Roger A. Jenkins \\ Chemical and Analytical Sciences and Computer Science and Mathematics ${ }^{\mathrm{a}}$ Division \\ Oak Ridge National Laboratory* \\ Oak Ridge, TN 37831-6120
}

\begin{abstract}
DISCLAIMER
This report was prepared as an account of work sponsored by an agency of the United States Government. Neither the United States Government nor any agency thereof, nor any of their employees, makes any warranty, express or implied, or assumes any legal liability or responsibility for the accuracy, completeness, or usefulness of any information, apparatus, product, or process disclosed, or represents that its use would not infringe privately owned rights. Reference herein to any specific commercial product, process, or service by trade name, trademark, manufacturer, or otherwise does not necessarily constitute or imply its endorsement, recommendation, or favoring by the United States Government or any agency thereof. The views and opinions of authors expressed herein do not necessarily state or reflect those of the United States Government or any agency thereof.
\end{abstract} *Managed by Lockheed Martin Energy Systems under U.S. Department of Energy contract
DE-AC05-84OR21400.

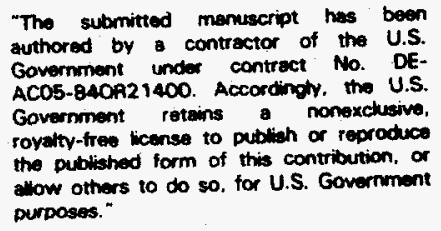


Reproducibility - The analysis of replicate samples within an assay is very reproducible. However, the reproducibility of the analysis of replicate test samples in different assays may be compromised if the integrity of the kit standards is not maintained.

Sensitivity - The detection limits for IAs are in the range of 0.1 to $2 \mathrm{ppm}$ in soil and lower in water. For soil, these detection limits are imposed by the background levels often found in soils and are not limited by IA technology..

Interferences - Soil matrix effects have been shown to have both positive and negative impacts on the results obtained by IA. Background immunoreactivity in the petroleum fuel hydrocarbon test caused the concentration in soils to be slightly over estimated. With the PAH test kits, extraction was less efficient from clay soils that from other types, resulting in an under estimation of PAHs in clay soils.

Time and cost effectiveness - Analysis by IA is relatively fast. It is very likely that a set of samples could be analyzed by IA in the time required to transport a similar set to a laboratory for analysis, and long before the results would come back from the laboratory. The advantages of on-site analysis for volatile and otherwise unstable analytes are obvious. The cost of analysis per sample is often significantly less for IA than for standard laboratory methods. Additional cost savings can be realized from the more rapid sample turn around afforded by IA analysis.

More detailed information about the performance of the IA-based test kits for mercury, PCBs and petroleum fuel hydrocarbons can be found in the following documents:

Waters, L.C., R.R. Smith, R.W. Counts, J.H. Stewart and R.A. Jenkins, Evaluation of field test kits including immunoassays for the detection of contaminants in soil and water. In "Field Screening Methods for Hazardous Wastes and Toxic Chemicals", Air and Waste Management Association, Pittsburgh, PA, vol 1, pp. 503-513 (1993).

Waters, L.C., R.A. Jenkins, R. Smith, J. Stewart and R. Counts, Immunoassay for mercury in soils: Method MB100. In "DOE Methods for Evaluating Environmental and Waste Management Samples", DOE, pp. MB100-1 to MB100-9 (1993).

Waters, L.C., R.A. Jenkins, R.R. Smith, R.W. Counts and J.H. Stewart, Immunoassay for polychlorinated biphenyls (PCBs) in soils: Method OSO20. In "DOE Methods for Evaluating Environmental and Waste Management Samples", DOE, pp. OSO20-1 to OSO20-17 (1993).

Waters, L.C., R.R. Smith, J.H. Stewart, R.A. Jenkins and R.W. Counts, Evaluation of two field screening test kits for the detection of PCBs in soil by immunoassay. J. AOAC International, 77: 1664$1671,1994$.

Waters, L.C., R.A. Jenkins, R.W. Counts and M.A. Palausky, Immunoassay for petroleum fuel hydrocarbons in soil: Method OS060. In "DOE Methods for Evaluating Environmental and Waste 


\section{Results of analyses for PCBs in soil samples using the EnSys immunoassay}

\begin{tabular}{|c|c|c|c|c|c|c|c|c|c|}
\hline \multirow[b]{4}{*}{$\begin{array}{c}\text { Sample } \\
\# \\
\end{array}$} & \multirow{4}{*}{$\begin{array}{c}\text { Reference } \\
\text { PCB } \\
\text { Conc. } \\
\text { (ppm) } \\
\end{array}$} & \multicolumn{8}{|c|}{ Photometer Reading and Interpretation } \\
\hline & & \multicolumn{4}{|c|}{ Analyst \#1 } & \multicolumn{4}{|c|}{ Analyst $\# 2$} \\
\hline & & \multicolumn{2}{|c|}{5 ppm Test Level } & \multicolumn{2}{|c|}{50 ppm Test Level } & \multicolumn{2}{|c|}{5 ppm Test Level } & \multicolumn{2}{|c|}{50 ppm Test Level } \\
\hline & & $\begin{array}{c}\text { Relative } \\
\text { Absorbance } \\
\end{array}$ & $\begin{array}{c}\text { Interpretation } \\
(\mathrm{ppm}) \\
\end{array}$ & $\begin{array}{c}\text { Relative } \\
\text { Absorbance } \\
\end{array}$ & $\begin{array}{c}\text { Interpretation } \\
\text { (ppm) }\end{array}$ & $\begin{array}{c}\text { Relative } \\
\text { Absorbance } \\
\end{array}$ & $\begin{array}{c}\text { Interpretation } \\
(\mathrm{ppm}) \\
\end{array}$ & $\begin{array}{c}\text { Relative } \\
\text { Absorbance } \\
\end{array}$ & $\begin{array}{c}\text { Interpretation } \\
(\mathrm{ppm}) \\
\end{array}$ \\
\hline 1 & 50 & -0.71 & 25 & -0.39 & 250 & -0.76 & 25 & -0.20 & 250 \\
\hline 1 & 50 & -0.75 & 25 & -0.50 & 250 & -0.69 & 3 & -0.32 & 250 \\
\hline 2 & 4 & -0.06 & 35 & 0.32 & $<50$ & -0.16 & 85 & 0.14 & 850 \\
\hline 3 & 10 & -0.16 & 25 & 0.32 & 85 & -0.29 & 25 & 0.00 & $250^{\circ}$ \\
\hline 3 & 10 & -0.36 & $\mathrm{~s}$ & -0.03 & $50^{\circ}$ & -0.45 & 25 & 0.30 & $<50$ \\
\hline 4 & 200 & -0.83 & 25 & -0.66 & 250 & -0.75 & 25 & -0.62 & 250 \\
\hline 5 & 40 & -0.83 & 25 & -0.27 & $\geqslant 50^{\circ}$ & -0.54 & 8 & -0.27 & $350^{\circ}$ \\
\hline 6 & 0 & 0.51 & $<5$ & 0.43 & $<50$ & 0.40 & 45 & 0.48 & $<50$ \\
\hline 6 & 0 & 0.16 & $<5$ & 0.36 & $<50$ & 0.24 & $<5$ & 0.47 & $<50$ \\
\hline 7 & 6 & -0.35 & 25 & 0.17 & $<50$ & -0.13 & 25 & 0.32 & $<50$ \\
\hline 8 & 80 & -0.81 & 25 & -0.29 & 250 & -0.83 & 25 & -0.53 & 250 \\
\hline 9 & 20 & -0.77 & 25 & -0.08 & $250^{\circ}$ & -0.66 & $\geq 5$ & -0.17 & $250^{\circ}$ \\
\hline 9 & 20 & -0.43 & $\mathrm{~s}$ & -0.07 & $250^{\circ}$ & -0.55 & 25 & 0.00 & $250^{\circ}$ \\
\hline 10 & 2 & 0.09 & 85 & 0.45 & 850 & 0.13 & $<5$ & 0.37 & $<50$ \\
\hline 10 & 2 & -0.08 & 85 & 0.22 & 850 & 0.30 & $<5$ & 0.36 & $<50$ \\
\hline 11 & 100 & -0.77 & 13 & -0.60 & $>50$ & -0.79 & 25 & -0.42 & 250 \\
\hline 11 & 100 & -0.67 & 25 & -0.28 & 250 & -0.82 & 25 & -0.82 & 250 \\
\hline 12 & 5 & -0.30 & 25 & 0.24 & $<50$ & -0.29 & 25 & 0.14 & $<50$ \\
\hline 12 & 5 & -0.18 & 25 & 0.24 & $<50$ & -0.40 & 25 & -0.29 & $250^{\circ}$ \\
\hline 13 & 60 & -0.66 & 85 & -0.18 & z 50 & -0.56 & 25 & -0.14 & 250 \\
\hline $14^{b}$ & $2.3 / 4.7$ & -0.14 & $\geq 5^{2}$ & 0.19 & $<50$ & -0.11 & $25^{\prime}$ & 0.42 & $<50$ \\
\hline $14^{b}$ & $2.3 / 4.7$ & -0.20 & $25^{3}$ & 0.28 & $<50$ & -0.04 & $25^{2}$ & 0.14 & $<50$ \\
\hline $15^{\mathrm{b}}$ & $11.1 / 11.5$ & -0.18 & $>5$ & -0.03 & $250^{\circ}$ & -0.40 & 25 & -0.09 & $250^{\circ}$ \\
\hline $16^{\mathrm{b}}$ & $72.5 / 83$ & -0.82 & $\geq 5$ & -0.49 & $\geq 50$ & -0.83 & $\geq 5$ & -0.26 & 250 \\
\hline 17 & 8 & -0.44 & 25 & 0.20 & $<50$ & -0.68 & 25 & -0.18 & $250^{\circ}$ \\
\hline
\end{tabular}

${ }^{\text {aF False positive result }}$ 'Field sample; reference concentration based on two analyses by GC 


\section{Results of analyses for PCBs in soil samples using the Millipore immunoassay}

\begin{tabular}{|c|c|c|c|c|c|c|c|c|c|}
\hline \multirow{2}{*}{\multicolumn{2}{|c|}{$\begin{array}{c}\text { Calibration } \\
\text { Standard Values }\end{array}$}} & \multirow{4}{*}{$\begin{array}{c}\text { Sample } \\
\# \\
\end{array}$} & \multirow{4}{*}{$\begin{array}{c}\text { Reference } \\
\text { PCB } \\
\text { Conc. } \\
\text { (ppm) } \\
\end{array}$} & \multicolumn{6}{|c|}{ Sample Absorbance Values and Interpretation of Results } \\
\hline & & & & & Analyst \#1 & & & Analyst $\not 2$ & \\
\hline Conc. & \multirow[b]{2}{*}{ Absorbance } & & & & & Interpretation & \multicolumn{2}{|c|}{ Absorbance } & \multirow{2}{*}{$\begin{array}{c}\text { Interpretatio } \\
\text { (ppm) }\end{array}$} \\
\hline$(\mathrm{nmm})$ & & & & Aliquot \#1 & Aliquot $\# 2$ & (ppm) & Aliquot :\#1 & Aliquot \#2 & \\
\hline & & \multirow[b]{2}{*}{ I } & \multirow[b]{2}{*}{80} & \multirow[b]{2}{*}{0.07} & \multirow[b]{2}{*}{0.08} & \multirow[b]{2}{*}{$>10$} & \multirow[b]{2}{*}{0.16} & \multirow[b]{2}{*}{0.14} & \multirow[b]{2}{*}{$>10$} \\
\hline 0 & $0.80,0.85$ & & & & & & & & \\
\hline 2 & $0.33,0.35$ & $2^{*}$ & 105 & 0.09 & 0.08 & $>10$ & 0.12 & 0.12 & $>10$ \\
\hline 10 & $0.13,0.24$ & 3 & 20 & 0.22 & 0.22 & $10^{\circ}$ & 0.27 & 0.31 & $>10$ \\
\hline & & $4^{2}$ & 16 & 0.26 & 0.25 & $2-10^{6}$ & 0.35 & 0.33 & $>10$ \\
\hline 0 & $1.35,1.39$ & 5 & 15 & 0.28 & 0.28 & $210^{6}$ & 0.30 & 0.30 & $>10$ \\
\hline 2 & $0.59,0.73$ & $6^{2}$ & 4,2 & 0.77 & 0.73 & $<2^{\mathbf{b}}$ & 0.87 & 0.91 & $<2^{+}$ \\
\hline 10 & $0.38,0.47$ & $7^{*}$ & $72.5 / 83$ & 0.16 & 0.14 & $>10$ & 0.20 & 0.19 & $>10$ \\
\hline & & & & & & & & & \\
\hline 0 & $1.20,1.17$ & $8^{a}$ & 32 & 0.17 & 0.17 & $>10$ & 0.23 & 0.24 & $>10$ \\
\hline 2 & $0.57,0.57$ & $9^{2}$ & $23 / 4.7$ & 0.43 & 0.43 & $2+10$ & 0.59 & 0.54 & $2-10$ \\
\hline 10 & $0.28,0.29$ & 10 & 50 & 0.15 & 0.15 & $>10$ & 0.16 & 0.15 & $>10$ \\
\hline & & 11 & 40 & 0.18 & 0.19 & $>10$ & 0.19 & 0.21 & $>10$ \\
\hline 0 & $1.44,1.43$ & 12 & 60 & 0.14 & 0.13 & $>10$ & 0.16 & 0.22 & $>10$ \\
\hline 2 & $0.76,0.76$ & 13 & 12 & 0.34 & 0.35 & $210^{\circ}$ & 0.37 & 0.33 & 310 \\
\hline 10 & $0.47,0.48$ & 14 & 0 & 1.57 & 1.62 & $<2$ & 1.73 & 1.65 & $<2$ \\
\hline & & & & & & & & & \\
\hline 0 & $1.09,1.14$ & $15^{\circ}$ & 1111115 & 0.28 & 0.30 & $10^{\mathrm{b}}$ & 0.32 & 0.29 & $>10$ \\
\hline 2 & $0.52,0.57$ & 16 & 30 & 0.17 & 0.17 & $>10$ & 0.20 & 0.21 & $>10$ \\
\hline 10 & $0.30,0.29$ & 17 & 5 & 0.50 & 0.48 & $2-10$ & 0.51 & 0.49 & 210 \\
\hline & & 18 & 100 & 0.11 & 0.09 & $>10$ & 0.10 & 0.11 & $>10$ \\
\hline 0 & $1.32,1.29$ & 19 & 10 & 0.45 & 0.38 & $2-10$ & 0.36 & 0.34 & 10 \\
\hline 2 & $0.60,0.64$ & 20 & 8 & 0.41 & 0.45 & $2-10$ & 0.43 & 0.39 & $2-10$ \\
\hline 10 & $0.34,0.35$ & 21 & 0 & 1.41 & 1.39 & $<2$ & 1.66 & 1.69 & $<2$ \\
\hline
\end{tabular}




\begin{tabular}{|c|c|c|c|c|c|}
\hline & \multicolumn{2}{|c|}{ BTEX standard values } & \multicolumn{3}{|c|}{ Test Samples } \\
\hline & BTEX (ppm) & $A_{450}$ & Concentration (ppm) & Asso & Interpretation (ppm) \\
\hline \multicolumn{6}{|l|}{ Exp.11 } \\
\hline & 0 & 0.94 & 0 & 0.80 & 0 to 2 \\
\hline & 2 & 0.77 & 2 & 0.71 & 2 to 12 \\
\hline & 12 & 0.36 & 5 & 0.55 & 2 to 12 \\
\hline & 60 & 0.18 & 10 & 0.42 & 2 to 12 \\
\hline & & & 20 & 0.25 & 12 to 60 \\
\hline & & & 30 & 0.19 & 12 to 60 \\
\hline & & & 50 & 0.16 & $>60^{\circ}$ \\
\hline & & & 100 & 0.10 & $>60$ \\
\hline \multicolumn{6}{|l|}{ Exp.:2 } \\
\hline & 0 & 0.84 & 0 & 0.84 & 0 \\
\hline & 2 & 0.62 & 2 & 0.63 & 0 to 2 \\
\hline & 12 & 0.33 & 5 & 0.52 & 2 to 12 \\
\hline & 60 & 0.13 & 10 & 0.43 & 2 to 12 \\
\hline & & & 20 & 0.32 & 12 to 60 \\
\hline & & & 30 & 0.26 & 12 to 60 \\
\hline & & & 50 & 0.17 & 12 to 60 \\
\hline & & & 100 & 0.10 & $>60$ \\
\hline
\end{tabular}

T indicates a false positive interpretation.

Analysis for gasoline in spiked soil samples using the Millipore immunoassay

\begin{tabular}{|c|c|c|c|c|c|}
\hline & \multicolumn{2}{|c|}{ BTEX standard values } & \multicolumn{3}{|c|}{ Teat Samples } \\
\hline & $\begin{array}{c}\text { BTEX/gasoline } \\
\text { (ppm) }\end{array}$ & A4so & $\begin{array}{c}\text { Gasoline } \\
\text { concentration } \\
\text { (ppm) }\end{array}$ & A 480 & $\begin{array}{c}\text { Interpretation } \\
\text { BTEX/gasoline } \\
\text { (ppon) }\end{array}$ \\
\hline \multicolumn{6}{|l|}{ Exp:11 } \\
\hline & $0 / 0$ & 1.27 & 80 & 0.18 & $>50(>58)$ \\
\hline & $2 / 2$ & 1.00 & 40 & 0.29 & 10 to 50 (11 to 58) \\
\hline & $10 / 11$ & 0.56 & 20 & 0.40 & 10 to 50 (11 to 58$)$ \\
\hline & $50 / 58$ & 0.28 & 10 & 0.55 & $-10(-11)$ \\
\hline & & & 5 & 0.71 & 2 to $10(2$ to 11$)$ \\
\hline & & & 1 & 1.10 & 0 to $2(0$ to 2$)$ \\
\hline \multicolumn{6}{|l|}{ Exp:112 } \\
\hline & 000 & 1.25 & 80 & 0.20 & $>50(-58)$ \\
\hline & $2 / 2$ & 0.88 & 40 & 0.31 & 10 to $50(11$ to 58$)$ \\
\hline & $10 / 11$ & 0.63 & 20 & 0.45 & 10 to 50 (11 to 58$)$ \\
\hline & $50 / 58$ & 0.26 & 10 & 0.55 & $>10^{\prime \prime}(-11)^{\prime}$ \\
\hline & & & 5 & 0.76 & 2 to $10(2$ to 11$)$ \\
\hline & & & 1 & 1.19 & 0 to 2 (0 to 2$)$ \\
\hline
\end{tabular}

$\rightarrow$ indicates a false positive interpretation. 
Analysis for BTEX in spiked soll samples using the Ohmicron immunoassay

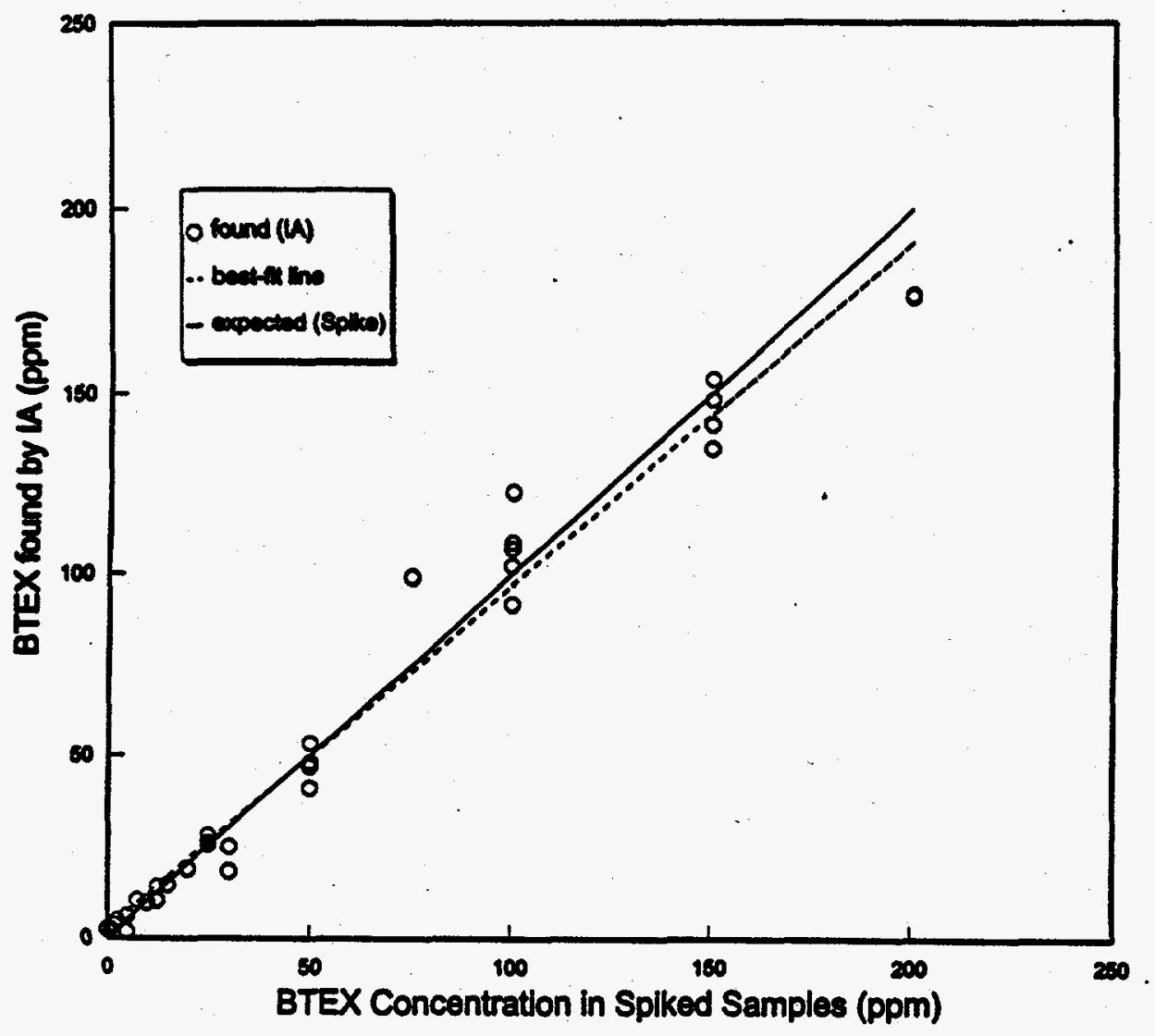

Analysis for gasoline in spiked soll using the Ohmicron immunoassay

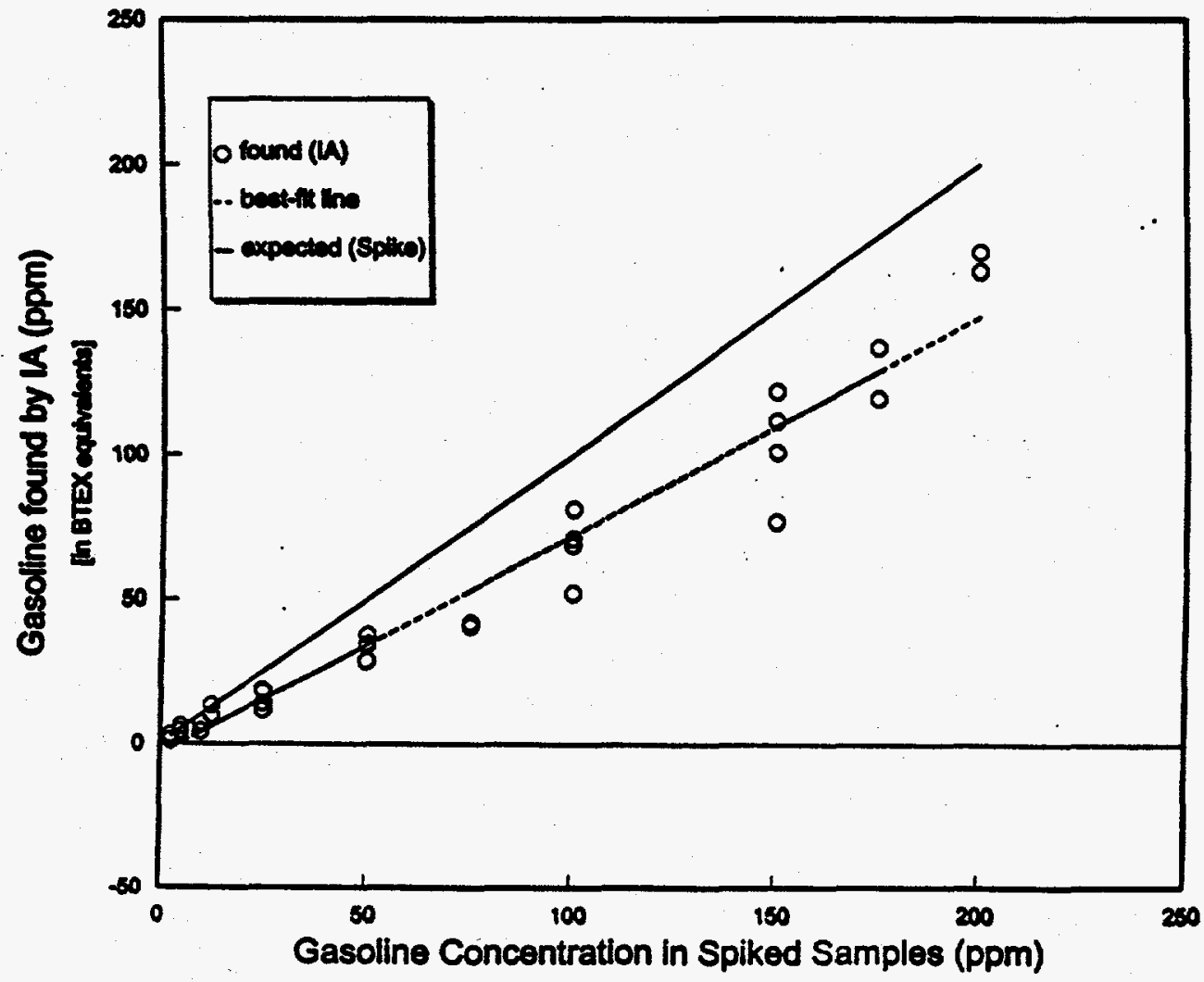


Analysis for PAH mixture in spiked soil samples using the Ohmicron immunoassay

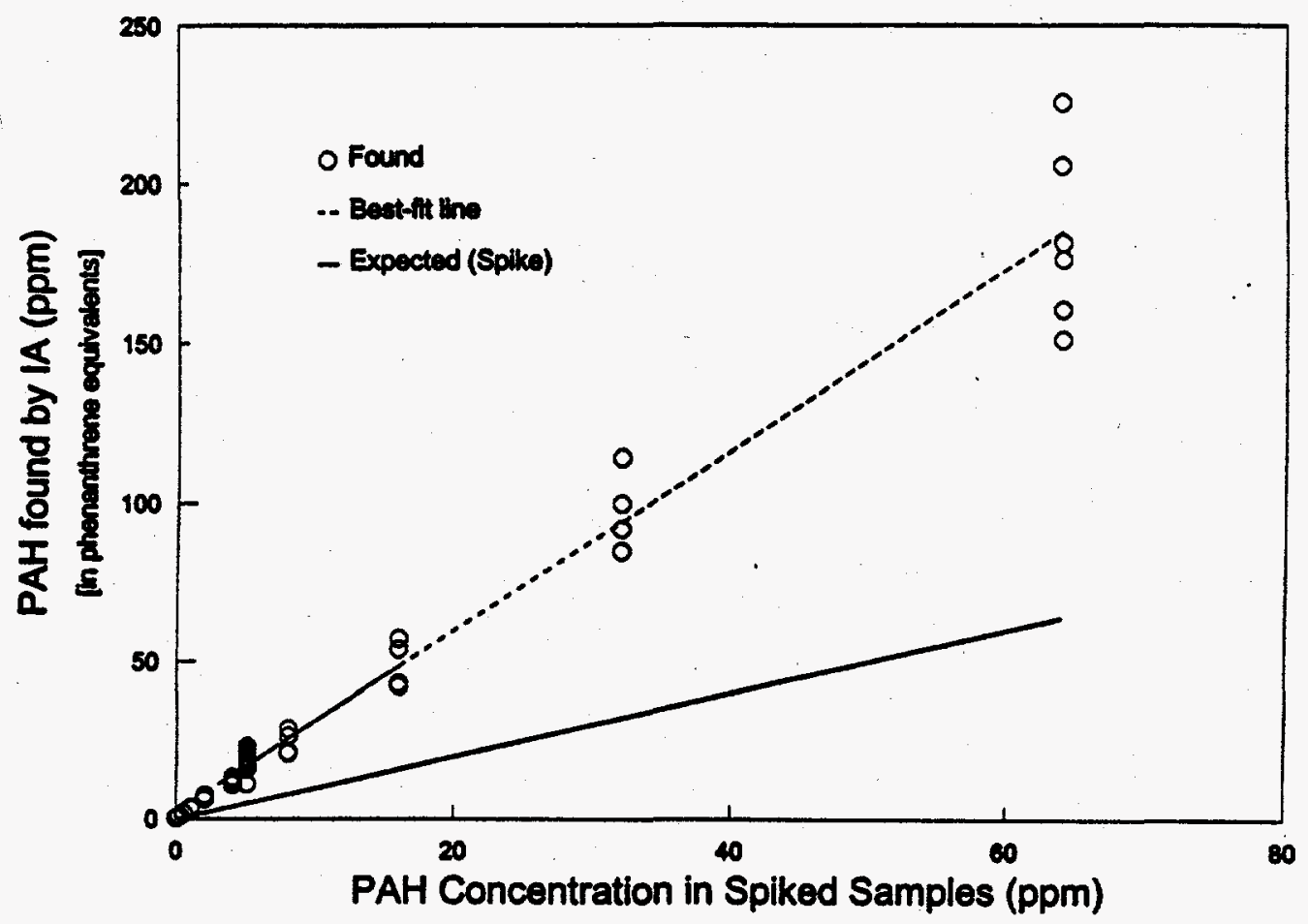

Analysis for creosote in spiked soil using the Ohmicron immunoassay

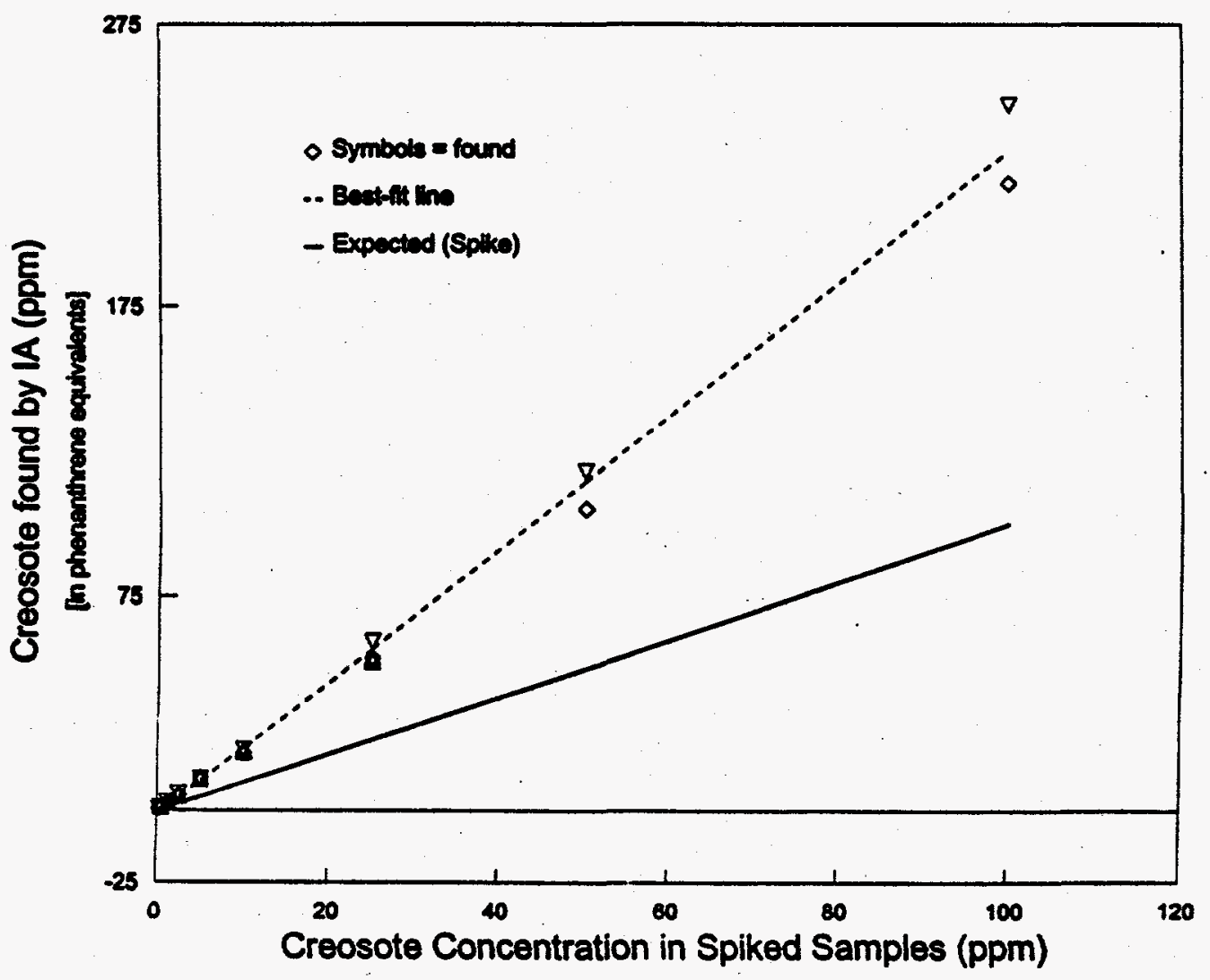




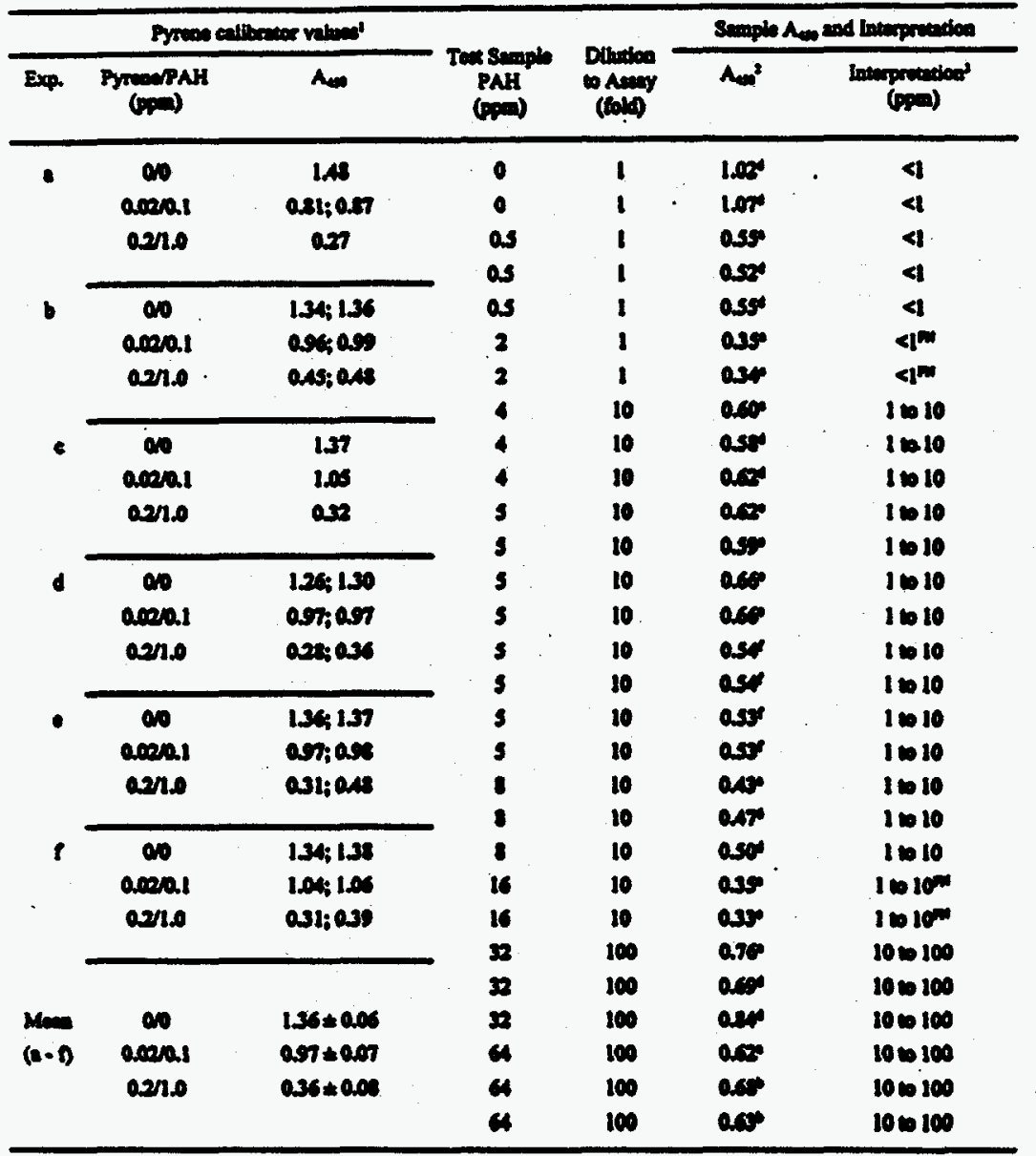

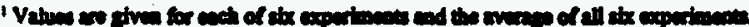

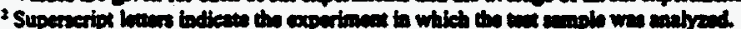

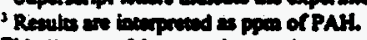

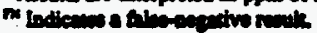

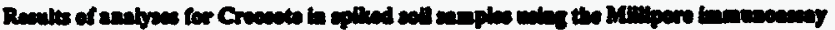

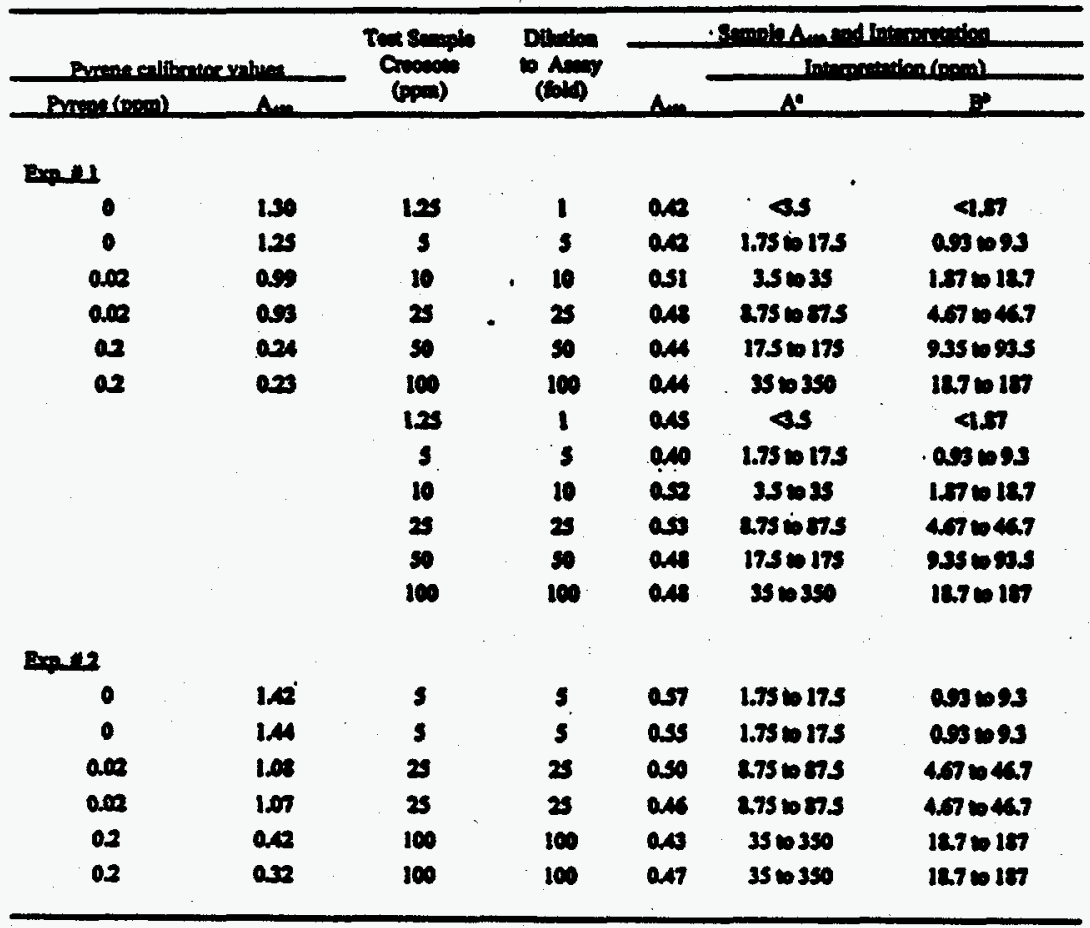

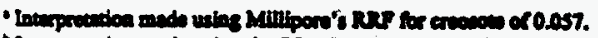

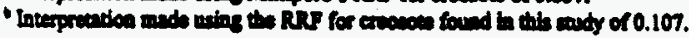




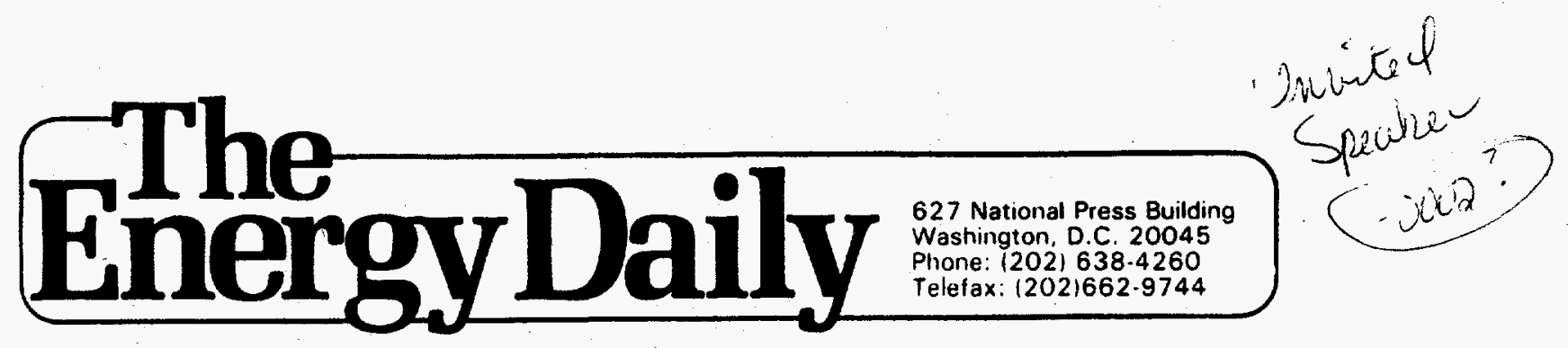

November 8, 1995

Dr. Larry Waters

Research Staff Member, Chemical \& Analytical Sciences Division

Oak Ridge National Laboratory

P.O. Box 2008

Oak Ridge, TN 37831-6120

Dear Larry:

I am writing to confirm your engagement at The Energy Daily conference On-Site Analysis: When Is It The Viable Option? to be held December 11-12, 1995. I enclose several conference brochures and a detailed agenda that includes all precise times.

Please note that the conference venue is the Crystal Gateway Marriott Hotel, 1700 Jefferson Davis Highway, Arlington, VA, 22202 tel: 703-920-3230. Note as well: we have you scheduled on Tuesday, December 12 at 10:00-10:30 am to give a 30 minute presentation titled Performance Of Immunoassay Kits For Site Characterization \& Remediation, allowing time for Q\&A to follow. I shall call you in late October to go over any points that we may need to discuss. In the meantime, your contact here is Courtney Smith on 202-662-9713.

Please complete the enclosed conference speaker questionnaire and return it to Courtney by November 22. Very important: To insure the success of our conference, we need your suggestions for people to receive our conference brochure, either to add to our mailing list or to provide you with additional brochures for any list that you may have. Please let us know on the questionnaire whether I should contact you or somebody else in your organization for names and addresses. Also, Courtney must have in advance a copy of your presentation to include in attendees' conference packets.

Thanks again for your participation. I hope you agree, we have a wonderful agenda and line-up of presenters. If you want to talk to me personally, please don't hesitate to call on 202-662-8563. I have voice mail.

Looking forward to working with you on the day, I remain,

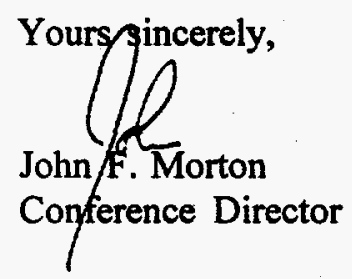

encls. 